\title{
Rendimiento de distintos sistemas de aprovechamiento en la corta de protección de un bosque de Nothofagus pumilio
}

\author{
Stocking and yield potential of three different logging \\ systems in a shelterwood cut of Nothofagus pumilio forest
}

\author{
Juan Manuel Cellini a*, Guillermo Martínez-Pastur b ${ }^{\text {, }}$ Carlos Spagarino c \\ Pablo Luis Peri ${ }^{\mathrm{d}}$, Ricardo Vukasovic ${ }^{\mathrm{e}}$ \\ *Autor de correspondencia: ${ }^{\text {a }}$ Universidad Nacional de La Plata, Laboratorio de Investigación de Sistemas Ecológicos y Ambientales \\ (LISEA), Diagonal 113 N 469 (B1904DPS) La Plata, República Argentina, tel/fax +54-221-4271442, cellini@gmail.com \\ ${ }^{\mathrm{b}}$ Centro Austral de Investigaciones Científicas (CONICET), Ushuaia, Argentina. \\ ${ }^{\mathrm{c}}$ Administración de Parques Nacionales, Laguna Blanca, Formosa, Argentina. \\ ${ }^{\mathrm{d}}$ INTA-UNPA-CONICET, Río Gallegos, Argentina. \\ ${ }^{\mathrm{e}}$ Consultora Servicios Forestales, Río Grande, Argentina.
}

\begin{abstract}
SUMMARY
Nothofagus pumilio is the most important timber native species of south Patagonia in Argentina and Chile. Total volume stock and timber production change according to site quality, occupation degree, growth development phases and management history of the stands. The aim of this work was to evaluate the yield of three different logging systems (short-logs, long-logs and full stems) under a shelterwood cut system of $N$. pumilio forest growing at a middle site quality stand in Tierra del Fuego (Argentina). Sample plots were obtained in an old-growth two strata stand (3.0 ha) with abundant regeneration (stand density index of 93.8-113.4 \%). Total volumes per hectare varied between 726.5 and $850.3 \mathrm{~m}^{3} \mathrm{ha}^{-1}$. The volume/basal area ratio varied from 11.8 to $12.1 \mathrm{~m}^{3} \mathrm{~m}^{-2}$. Yield indexes obtained were $45.5 \%$ for full stems, $21.3 \%$ for long-logs and $22.4 \%$ for short-logs logging systems. A model performed to estimate the timber volume for each system shows that full stems is significantly different from the other two. Several studies related to timber production were compared and discussed. Thus, applying full stems system results in a higher timber volume. Considering new alternatives in the planning of $N$. pumilio forests allows for higher harvest rates, increasing profits for the forestry company and minimizing the damage to the forest.
\end{abstract}

Key words: forest harvesting, log volume, biometric models, volume yield, forest structure, lenga, Patagonia.

\section{RESUMEN}

Los bosques de Nothofagus pumilio constituyen la masa forestal de mayor importancia económica del sur de Argentina y Chile. Las existencias de volumen total y el rendimiento volumétrico varían de acuerdo a la calidad de sitio, el grado de ocupación, la fase de crecimiento y la historia forestal del rodal. El objetivo de este trabajo fue evaluar las existencias y el potencial productivo en cantidad y calidad de productos para la industria del aserrado, mediante tres sistemas de aprovechamiento (trozas cortas, trozas largas y fustes completos) en la corta de protección de un bosque de N. pumilio de calidad de sitio III en Tierra del Fuego (Argentina). Los ensayos se llevaron a cabo en un bosque maduro irregular con dos estratos y abundante regeneración (3,0 ha; IDR 93,8-113,4 \%). Los volúmenes totales variaron entre 726,5 y $850,3 \mathrm{~m}^{3} \mathrm{ha}^{-1}$, presentando una relación volumen/área basal de 11,8 a $12,1 \mathrm{~m}^{3} \mathrm{~m}^{-2}$. Los índices de aprovechamiento obtenidos fueron: $45,5 \%$ para fustes completos, $21,3 \%$ para trozas largas y $22,4 \%$ para trozas cortas. Con un modelo se estimó el volumen maderable para cada sistema, donde fustes completos resultó significativo en mayor volumen maderable. Considerar nuevas alternativas en la planificación del aprovechamiento dentro del manejo forestal para bosques de N. pumilio, como el sistema de fustes completos, permite obtener mayores índices de cosecha, aumentando los beneficios para la empresa forestal y minimizando el daño en el bosque, debido al menor recorrido de la maquinaria en el aprovechamiento forestal.

Palabras clave: aprovechamiento forestal, volumen aprovechable, rendimiento volumétrico, estructura, lenga, Patagonia.

\section{INTRODUCCIÓN}

Los bosques de Nothofagus pumilio (Poepp. et Endl.) Krasser (lenga) constituyen la masa forestal de mayor im- portancia económica en superficie y volumen del sur de Argentina y Chile (Collado 2001, Martínez Pastur y Lencinas 2005). Nothofagus pumilio es una especie semiheliófila que presenta una instalación promedio de 623.100 
renovales por hectárea en elevadas coberturas forestales (Martínez Pastur et al. 2011), requiriendo mayor disponibilidad de luz para poder desarrollarse (Martínez Pastur et al. 2007a). El método de regeneración empleado que se adapta a esta especie es el de cortas de protección, que consiste en la apertura gradual del dosel para permitir el desarrollo de la regeneración. Esta alternativa propone la transformación del bosque virgen en un sistema regular que permita obtener aumentos en el crecimiento, estado sanitario y madera de calidad, mejorando los índices de aprovechamiento y su posterior rendimiento en el aserradero (Schmidt y Urzúa 1982, Martínez Pastur et al. 2000).

En Tierra del Fuego existen 215.000 ha de bosques productivos de N. Pumilio (Collado 2001). Las existencias de volumen total con corteza varían entre 300 y $1.300 \mathrm{~m}^{3}$ $\mathrm{ha}^{-1}$ de acuerdo a su calidad de sitio, grado de ocupación, fase de crecimiento e historia forestal (Martínez Pastur et al. 2000). Las fases de crecimiento son etapas del ciclo de desarrollo natural del bosque de Nothofagus spp. de duración variable, las cuales se asocian a rangos de edades y a estructuras particulares (Schmidt y Urzúa 1982). Estas etapas comienzan con la de crecimiento óptimo inicial, caracterizada por poseer una corteza lisa a lo largo del tronco, luego el crecimiento óptimo final donde la corteza comienza a resquebrajarse. En la fase de envejecimiento la corteza se encuentra agrietada y formando placas. La fase de desmoronamiento se caracteriza por desprendimiento de la corteza y hendiduras profundas a lo largo del tronco (Schmidt y Urzúa 1982). Para estos bosques existe una clasificación de clases de sitio (Martínez Pastur et al. 1997) que presenta cinco calidades (I a V) definidas con fines de uso forestal en Patagonia Sur, siendo la calidad de sitio el principal factor que afecta al rendimiento volumétrico del rodal (Martínez Pastur et al. 2000). La mayoría de los bosques productivos de Tierra del Fuego se desarrollan en una clase de sitio III y a la madurez poseen una altura dominante que varía entre 20,5 y $24,0 \mathrm{~m}\left(\mathrm{IS}_{60}=13,15\right.$ 16,50 m; Martínez Pastur et al. 1997).

Desde el comienzo de las actividades forestales en Tierra del Fuego (Argentina), la práctica tradicional de aprovechamiento ha consistido en el apeo de los mejores individuos (floreo) (Gea-Izquierdo et al. 2004). Como resultado los bosques intervenidos presentan individuos remanentes sobremaduros y de baja calidad. Sin embargo, en los rodales de mejor calidad de sitio se realizaron cortas intensivas, llegando a la tala rasa de importantes superficies cuando se extrajo leña en las inmediaciones de centros urbanos (Mosqueda 1995, Gea-Izquierdo et al. 2004). En la actualidad, con la aplicación de la corta de protección, parte de los árboles son marcados como dosel de protección y otra parte no es aprovechada debido a un bajo rendimiento, dejando como resultado una corta incompleta en este método de regeneración. En la década del 90, la demanda de madera en el mercado local se basó en las grandes escuadrías (tablones, tablas y tirantes), siendo necesario realizar una alta selección de trozas (4-6 m de largo, diámetros superiores a los $35 \mathrm{~cm}$ y buena sanidad) del tipo "A" y "B" según la clasificación propuesta por Cordone y Bava (1997). El sistema de aprovechamiento tradicional consiste en el volteo, corte basal sanitario o desculate (corte realizado en la base del árbol apeado para eliminar las irregularidades en el tronco debido al apeo y pudriciones en la primera troza) y producción de trozas de dimensiones fijas en el bosque. Este sistema tiene como inconvenientes las malas condiciones de trabajo del motosierrista (ya que al seleccionar en el árbol apeado las trozas es necesario hacer varios cortes entre los residuos), una alta selección en la calidad de trozas (se aprovechan las trozas de mayor calidad), la subutilización del rendimiento del tractor articulado (skidder) (generalmente la limitante es el número de lingaso estrobos y no el volumen a maderear) y un aumento en la pérdida de trozas (10\% al $20 \%$ del volumen generado) durante el madereo por tener difícil acceso o por encontrarse entre los residuos (Gea-Izquierdo et al. 2004). Al ser limitadas este tipo de trozas en el bosque (5\% y $20 \%$, respectivamente), se obtienen por medio del sistema tradicional de aprovechamiento un bajo porcentaje de corta y un índice de cosecha del $5 \%$ al $10 \%$ (volumen de trozas/volumen total del bosque), con volú-

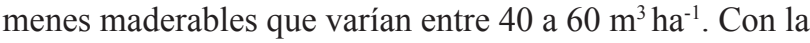
mejora tecnológica en los aserraderos, se incorporaron en la producción las trozas de menor calidad (trozas "C", con defectos localizados o mala forma) y diámetros en punta fina mayores a $20 \mathrm{~cm}$. Esta menor calidad en las trozas determina la necesidad de comparar el rendimiento volumétrico entre el sistema de aprovechamiento tradicional con otros sistemas para evaluar la calidad y cantidad de trozas aprovechadas. El objetivo de este trabajo es evaluar las existencias y el potencial productivo en cantidad y calidad de productos para la industria del aserrado y de síntesis, mediante tres sistemas de aprovechamiento (trozas cortas, trozas largas y fustes completos) en la corta de protección de un bosque de $N$. pumilio de calidad de sitio III en Tierra del Fuego (Argentina).

\section{MÉTODOS}

Área bajo estudio. Se trabajó en un bosque puro de N. pumilio en la estancia San Justo, Tierra del Fuego

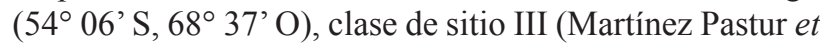
al. 1997), donde el aserradero Los Castores realiza el aprovechamiento forestal de acuerdo a la reglamentación de la ley forestal provincial $\mathrm{N}^{\circ} 145$, utilizando como método de reproducción monte alto bajo cubierta protectora (corta de protección) (Schmidt y Urzúa 1982).

Caracterización del bosque. Para este estudio se seleccionó al azar una superficie de 3 ha en un bosque sin intervención (BSI) donde se realizaron 15 parcelas contiguas de 40 x $50 \mathrm{~m}$. En cada una de ellas se caracterizó la estructura del bosque por medio de la altura dominante (100 árboles más altos por hectárea), área basal, diámetro cuadrático 
medio (DCM), número de árboles por hectárea, número de renovales por hectárea ( 1 a $10 \mathrm{~cm}$ DAP), clase social o de copas (dominante, codominante, intermedio o suprimido) y fase de crecimiento de los individuos (crecimiento óptimo inicial, crecimiento óptimo final, envejecimiento o desmoronamiento) (Schmidt y Urzúa 1982).

Marcación y volteo. En la superficie seleccionada se realizó una marcación de los árboles semilleros de acuerdo al tratamiento de corta de protección, dejando en pie un $40 \%$ del área basal original con un distanciamiento máximo entre árboles de $9 \mathrm{~m}$. Los árboles semilleros seleccionados debían tener copa amplia, ser dominantes y bajo coeficiente de esbeltez debido a que se seleccionan los árboles de menor susceptibilidad a daños por viento y nieve (Cremer et al. 1982, Wilson y Oliver 2000).

El volteo se realizó con personal capacitado mediante el uso de motosierras. Se evaluaron dos tipos de cortas en las 3 ha seleccionadas del bosque sin intervención (BSI): la primera consistió en el volteo de los individuos de mejor calidad de acuerdo al criterio del motosierrista (FL), sin cortar los árboles semilleros (este tipo de corta, similar al floreo, es tradicionalmente aplicado en la región), mientras que en una segunda etapa se completó la corta de protección de acuerdo a la marcación realizada $(\mathrm{CP})$. Se midió el volumen de todos los árboles volteados (volumen total con y sin corteza) incluyendo el tocón, ramas y fuste hasta $1 \mathrm{~cm}$ de diámetro. La determinación de los volúmenes parciales se realizó mediante la fórmula de Smalian (Cailliez 1980, Clutter et al. 1983).

Sistemas de aprovechamiento. Se trabajó en las 3 ha seleccionadas con tres sistemas de aprovechamiento en cinco parcelas contiguas de $40 \times 50 \mathrm{~m}$. cada uno. En el primer tratamiento se aplicó un sistema de aprovechamiento de volteo y trozado, consistiendo el producto en trozas cortas (TC) para aserrado de 3 a $5 \mathrm{~m}$ de longitud y un diámetro en punta fina superior a $20 \mathrm{~cm}$, no debiendo tener más de un tercio de pudrición en la cara más afectada, sin encorvaduras en el eje longitudinal (flecha), ni defectos generalizados (trozas A, B y C). En el segundo tratamiento se extrajeron trozas sin restricción de longitud (TL), con las mismas características que el tratamiento anterior, pero con menos exigencias respecto a la flecha y las características externas de las trozas. En un último tratamiento se realizó una extracción de fustes completos $(\mathrm{F})$, con un corte en la base del árbol y otro hasta un diámetro de $20 \mathrm{~cm}$ en el fuste. En este tratamiento, el trozado de los fustes se realizó con motosierra en la cancha de acopio.

Caracterización del volumen. El volumen se separó en maderable (fustes, trozas cortas y largas), astillable y desecho. Las trozas astillables tuvieron una longitud de $2,44 \mathrm{~m}$ y un diámetro mínimo con corteza de $10 \mathrm{~cm}$, no debiendo tener más de dos tercios de pudrición en la peor cara. Como desecho se consideró todo material comercialmen- te inutilizable ( $\sin$ tener en cuenta la venta de leña). Para el cálculo del volumen de trozas se utilizó la fórmula de Smalian. En el caso de fustes se utilizó la fórmula de Newton debido a sus grandes dimensiones (Husch et al. 2003). El volumen total y aprovechable del dosel de protección se caracterizó mediante las funciones propuestas por Martínez Pastur (2006), que incorpora la clase de sitio como variable.

Estimación en pie del volumen aprovechable. Para comparar el rendimiento volumétrico los árboles remanentes del dosel de protección se realizó una estimación del volumen de trozas en pie mediante el modelo [1] (Martínez Pastur et al. 2001, 2002):

$$
\operatorname{VMSC}\left(\mathrm{m}^{3}\right)=\mathrm{M} \times \mathrm{I}
$$

Donde, "M" = modelo no lineal que predice el volumen maderable (VMSC) sin corteza de árboles individuales, e "I" = índice de discriminación que presenta un valor 0 o 1 $(0=$ no maderable; $1=$ maderable $)$ generado con base en clasificaciones empíricas.

El valor del índice "I" se obtiene de una tabla en donde se utilizan las variables clase social (CS) (S-suprimido, Iintermedio, C-codominante y D-dominante), fase de desarrollo del árbol (COI-crecimiento óptimo inicial, COFcrecimiento óptimo final, ENV-envejecimiento, DM-desmoronamiento) según Schmidt y Urzúa (1982), y el DAP en cm para su obtención. El componente "M" representa el volumen maderable sin corteza que aportará un árbol cuyo componente I sea igual a 1. El modelo M quedó representado por la siguiente ecuación ajustada mediante técnicas de regresión no lineal:

$$
\mathrm{M}=\mathrm{a} \times \mathrm{DAP}^{\mathrm{b}}
$$

Donde, a y $\mathrm{b}=$ parámetros de la ecuación y $\mathrm{DAP}=$ diámetro a $1,3 \mathrm{~m}(\mathrm{~cm})$

Análisis estadístico. Se realizó un análisis de varianza para analizar el efecto de los distintos tratamientos de aprovechamiento (TC, TL y F) sobre variables de estructura forestal (DCM, área basal, altura dominante, densidad y porcentaje de individuos para cada CS y fase de desarrollo del árbol) mediante la prueba de F, utilizando la prueba de Tukey para la comparación de las medias.

El nivel de significancia utilizado para todos los casos fue de $\alpha=0,05$. Se realizó una comparación del componente " $M$ " del modelo de volumen de trozas en pie para los tratamientos TC, TL y F. El ajuste de todos los modelos se llevó a cabo mediante técnicas de regresión no lineal. Para la estimación de los parámetros se utilizó el algoritmo propuesto por Marquardt (1963). La evaluación estadística de los modelos se llevó a cabo a través del coeficiente de determinación ajustado $\left(\mathrm{r}^{2}\right)$ y el error absoluto promedio. 
Para evaluar el comportamiento del discriminante "I" de la estimación del volumen de trozas en pie frente a la muestra $(0=$ no maderable; $1=$ maderable $)$ se analizaron las frecuencias de individuos que podrían aportar trozas y que no podrían aportar trozas, realizando una prueba de Chicuadrado entre las frecuencias observadas y calculadas, discriminando por clase social y fase de crecimiento.

\section{RESULTADOS}

Caracterización de la estructura inicial y remanente bajo diferentes tipos de corta. El bosque intervenido presentó la estructura de un bosque maduro irregular, con dos estratos bien diferenciados y abundante regeneración por bosquetes (cuadro 1). El bosque pertenece a la clase de sitio III (según la clasificación propuesta por Martínez Pastur et al. 1997), con alturas dominantes que fluctúan entre los 22 y $23 \mathrm{~m}$. Los valores de densidad promedio variaron entre un $93,8 \%$ y un $113,4 \%$ de acuerdo al índice de densidad de Reineke propuesto por Martínez Pastur (2006), lo que indica que los rodales se encontraban en el máximo grado de ocupación (59,7 a 73,3 m² de área basal por hectárea). Estos rodales se pueden clasificar como bosques en fase de envejecimiento (40,5\% al 67,1 \% de los árboles se encuentran en dicha fase), con un bajo porcentaje de individuos en fase de desmoronamiento $(4,6 \%$ al $9,1 \%)$ que en su mayoría fueron dejados como dosel protector luego de la corta. Este tipo de estructura es la más representativa de los bosques vírgenes productivos de Tierra del Fuego, por su calidad de sitio y su alta proporción de individuos maderables (Martínez Pastur et al. 1997). Al analizar la estructura original entre los tratamientos de diferentes sistemas de aprovechamiento, se detectaron diferencias significativas en el número de árboles en la fase de crecimiento óptimo final y número de árboles intermedios en el tratamiento de fuste completo respecto de los otros dos (cuadro 1). Si bien no hay diferencias significativas, se observa que el tratamiento $\mathrm{F}$ (fustes completos) tiene mayor número de árboles (33-35\%), con mayor número de individuos en fase de crecimiento óptimo final (19-28 \%) y menos individuos codominantes (5-16\%). Estas diferencias deberán ser tenidas en cuenta al analizar el rendimiento volumétrico por hectárea. No se han encontrado interacciones significativas en los análisis de varianza con lo que se puede señalar la independencia de los tratamientos.

En el análisis de la varianza de la estructura del floreo (FL) se detectaron diferencias significativas en gran parte de las variables estudiadas (cuadro 1). En este sistema de aprovechamiento se apeó un $23 \%$ del área basal original en los tratamientos de trozas cortas y trozas largas, y un $50 \%$ del área basal original en el tratamiento de fustes completos, aprovechando árboles entre 30-70 cm de dap, con un promedio de $41-48 \mathrm{~cm}$. Se voltearon entre 77 y 274 árboles lo que representó la eliminación del 19 al $50 \%$

Cuadro 1. Análisis de varianza de la estructura del bosque bajo estudio en los diferentes tratamientos.

Analyses of variance of the forest structure in the three logging systems.

\begin{tabular}{cccccccccccccc}
\hline Tratamiento & DCM & AB & HD & Densidad & Densidad $<10$ & COI & COF & ENV & DM & S & I & C & D \\
\hline SITC & 43,2 & 59,69 & 22,91 & 411 & 31 & 11,7 & $16,6 \mathrm{a}$ & 67,1 & 4,6 & 24,8 & $19,0 \mathrm{a}$ & 42,3 & 13,9 \\
SITL & 44,5 & 63,35 & 22,25 & 415 & 20 & 3,6 & $26,1 \mathrm{a}$ & 62,1 & 8,2 & 19,8 & $16,4 \mathrm{a}$ & 53,2 & 10,6 \\
SIF & 41,0 & 73,29 & 21,84 & 555 & 5 & 5,4 & $45,0 \mathrm{~b}$ & 40,5 & 9,1 & 18,9 & $30,6 \mathrm{~b}$ & 36,9 & 13,6 \\
FLTC & $47,7 \mathrm{~b}$ & $13,80 \mathrm{a}$ & --- & $77 \mathrm{a}$ & 0 & $0,0 \mathrm{a}$ & $3,9 \mathrm{a}$ & $93,5 \mathrm{ab}$ & 2,6 & $1,3 \mathrm{a}$ & $10,4 \mathrm{a}$ & $72,7 \mathrm{~b}$ & 15,6 \\
FLTL & $46,0 \mathrm{~b}$ & $14,47 \mathrm{a}$ & --- & $87 \mathrm{a}$ & 0 & $0,0 \mathrm{a}$ & $5,8 \mathrm{a}$ & $91,9 \mathrm{~b}$ & 2,3 & $1,1 \mathrm{a}$ & $1,2 \mathrm{a}$ & $87,4 \mathrm{~b}$ & 10,3 \\
FLF & $41,0 \mathrm{a}$ & $36,18 \mathrm{~b}$ & --- & $274 \mathrm{~b}$ & 2 & $3,7 \mathrm{~b}$ & $54,7 \mathrm{~b}$ & $39,4 \mathrm{a}$ & 2,2 & $20,2 \mathrm{~b}$ & $32,2 \mathrm{~b}$ & $46,7 \mathrm{a}$ & 10,9 \\
CPTC & 37,5 & 35,53 & --- & 321 & $31 \mathrm{~b}$ & 6,2 & $27,7 \mathrm{a}$ & 58,3 & 7,8 & $22,8 \mathrm{a}$ & $24,9 \mathrm{a}$ & 46,7 & 5,6 \\
CPTL & 38,5 & 38,75 & --- & 332 & $20 \mathrm{ab}$ & 5,4 & $32,5 \mathrm{ab}$ & 56,6 & 5,5 & $25,0 \mathrm{a}$ & $21,7 \mathrm{a}$ & 45,8 & 7,5 \\
CPF & 39,2 & 42,93 & --- & 385 & $3 \mathrm{a}$ & 11,1 & $41,3 \mathrm{~b}$ & 43,3 & 4,5 & $24,4 \mathrm{a}$ & $27,1 \mathrm{~b}$ & 44,4 & 8,1 \\
RTC & $58,5 \mathrm{~b}$ & $24,17 \mathrm{a}$ & --- & $90 \mathrm{a}$ & 0 & 0,0 & $4,5 \mathrm{a}$ & 94,4 & $1,1 \mathrm{a}$ & 0,0 & $8,9 \mathrm{a}$ & 55,5 & 35,6 \\
RTL & $61,4 \mathrm{~b}$ & $24,60 \mathrm{a}$ & --- & $83 \mathrm{a}$ & 0 & 0,0 & $0,0 \mathrm{a}$ & 83,1 & $16,9 \mathrm{ab}$ & 0,0 & $2,3 \mathrm{a}$ & 62,7 & 35,0 \\
RF & $46,6 \mathrm{a}$ & $28,97 \mathrm{~b}$ & ---- & $170 \mathrm{~b}$ & 2 & 0,0 & $34,2 \mathrm{~b}$ & 47,0 & $18,8 \mathrm{~b}$ & 18,8 & $23,5 \mathrm{~b}$ & 35,3 & 22,4 \\
\hline
\end{tabular}

$\mathrm{BSITC}=$ situación inicial tratamiento de trozas cortas; BSITL = situación inicial tratamiento de trozas largas; BSIF = situación inicial tratamiento de fustes completos; FLTC = floreo tratamiento de trozas cortas FLTL = floreo tratamiento de trozas largas; FLF = floreo tratamiento de fustes completos; $\mathrm{CPTC}=$ corta de protección tratamiento de trozas cortas; $\mathrm{CPTL}=$ corta de protección tratamiento de trozas largas; $\mathrm{CPF}=$ corta de protección tratamiento de fustes completos; $\mathrm{RTC}=$ bosque remanente tratamiento de trozas cortas; $\mathrm{RTL}=$ bosque remanente tratamiento de trozas largas; $\mathrm{RF}=$ bosque remanente tratamiento de fustes completos; $\mathrm{DCM}=$ diámetro cuadrático medio $(\mathrm{cm}) ; \mathrm{AB}=$ área basal $\left(\mathrm{m}^{2} \mathrm{ha}^{-1}\right) ; \mathrm{HD}=$ altura dominante $(\mathrm{m}) ;$ Densidad = número de árboles por hectárea $\left(\mathrm{n} \mathrm{ha}^{-1}\right) ; \mathrm{COI}=$ crecimiento óptimo inicial (\%); $\mathrm{COF}=$ crecimiento óptimo final (\%); ENV = envejecimiento (\%); DM = desmoronamiento (\%); $\mathrm{S}=$ suprimido (\%); I = intermedio (\%); $\mathrm{C}=$ codominante (\%); $\mathrm{D}=$ dominante (\%). En cada columna, letras diferentes indican diferencias significativas $(P<0,01)$. 
de los individuos. Más del $90 \%$ de los árboles estaba en fase de envejecimiento (tratamientos FLTC y FLTL), que son los individuos que aportan el mayor porcentaje de madera sana aserrable (Martínez Pastur et al. 2009), siendo en su gran mayoría codominantes (buena forma de fuste con copas amplias y sin daños externos). El tratamiento de floreo con fustes completos (FLT) mostró diferencias en la fase de crecimiento y clase social de los árboles apeados, observándose un mayor número de árboles volteados y en que el porcentaje de los crecimientos óptimos finales, intermedios y suprimidos fue significativamente mayor en FLT que en los tratamientos de FLTC y FLTL.

Con la aplicación del tratamiento de $\mathrm{CP}$ el bosque presentó una estructura más homogénea, al dejar de observarse las diferencias encontradas en el análisis de varianza del tratamiento de floreo en las variables de $\mathrm{DCM}, \mathrm{AB}$, densidad, fase de crecimiento y clase social. Cuando se completó la corta del $60 \%$ del área basal, el 70-80\% de los individuos fueron apeados. En esta segunda etapa se aprovecharon principalmente árboles en crecimiento óptimo final, desmoronamiento y algunos en fase de envejecimiento no apeados durante el floreo; siendo en su gran mayoría intermedios o dominantes.

El dosel de protección (40\% del área basal original) quedó conformado por árboles de buen porte $(46,6$ a $58,5 \mathrm{~cm}$ DCM) en fase de envejecimiento-desmoronamiento (65-100\% de los individuos) de clase social codominante-dominante (57-98\% de los individuos).

Estimación en pie del volumen aprovechable. Los valores observados volumen maderable sin corteza - DAP presentan una distribución heterogénea y dispersa debido a la presencia de árboles no maderables, y diferentes sistemas de aprovechamiento. La modelización del volumen maderable sin corteza se dificulta al presentar valores nulos $(\mathrm{VTSC}=0)$ a lo largo del gradiente diamétrico. Para poder discriminar los árboles que presentaron volumen maderable sin corteza se ajustó el modelo (1) en donde se aplica un discriminante "I" para separar aquellos que aportan volumen de trozas ( $(=1)$ de los que no presentan volumen de trozas $(I=0)$. En el cuadro 2 se define la clave de discriminantes, en donde la construcción del componente I se realizó analizando las probabilidades de encontrar individuos que aportaran o no volumen maderable sin corteza. Se evaluó la calidad del discriminante efectuando una prueba de Chi-cuadrado entre las frecuencias observadas y las modeladas, resultando adecuado ya que no se observaron diferencias significativas (cuadro 3).

La inclusión del DAP como variable independiente explicó entre el 70 y el $80 \%$ de la variación encontrada en los datos, siendo este ajuste adecuado, considerando que la variable dependiente presenta un comportamiento muchas veces errático y de difícil explicación. Los modelos ajustados para cada sistema de aprovechamiento quedaron definidos por los parámetros y estadísticos que se presentan en el cuadro 4. Los modelos presentaron un bajo error absoluto promedio en la predicción del volumen maderable sin corteza. Para el sistema de aprovechamiento por fuste completo se observó que el modelo difiere significativamente de los modelos volumen maderable sin cortezatroza corta y volumen maderable sin corteza-troza larga al comparar los intervalos de confianza de los parámetros, resultando en un mayor volumen maderable al aplicar este sistema. En la figura 1 se muestra que un árbol aprovechable de $40 \mathrm{~cm}$ de DAP genera un $50 \%$ más de volumen maderable que un árbol del mismo diámetro para el sistema troza corta o troza larga.

Cuadro 2. Componente "I" del modelo (1) de estimación del volumen aprovechable en pie.

Parameter "I" from (1) model of log under bark volume estimation

\begin{tabular}{|c|c|c|c|}
\hline Fase de crecimiento del árbol & Clase social & $\operatorname{DAP}(\mathrm{cm})$ & I \\
\hline \multirow[t]{5}{*}{ Crecimiento óptimo inicial } & Suprimido & & 0 \\
\hline & Intermedio & $<30$ & 0 \\
\hline & Intermedio & $\geq 30$ & 1 \\
\hline & Codominante & $<30$ & 0 \\
\hline & Codominante & $\geq 30$ & 1 \\
\hline \multirow[t]{7}{*}{ Crecimiento óptimo final } & Suprimido & $<25$ & 0 \\
\hline & Suprimido & $\geq 25$ & 1 \\
\hline & Intermedio & $<25$ & 0 \\
\hline & Intermedio & $\geq 25$ & 1 \\
\hline & Codominante & $<30$ & 0 \\
\hline & Codominante & $\geq 30$ & 1 \\
\hline & Dominante & & 1 \\
\hline \multirow[t]{9}{*}{ Envejecimiento } & Suprimido & & 0 \\
\hline & Intermedio & $<35$ & 0 \\
\hline & Intermedio & $\geq 35<40$ & 1 \\
\hline & Intermedio & $\geq 40$ & 0 \\
\hline & Codominante & $<35$ & 0 \\
\hline & Codominante & $\geq 35<55$ & 1 \\
\hline & Codominante & $\geq 55$ & 0 \\
\hline & Dominante & $<65$ & 1 \\
\hline & Dominante & $\geq 65$ & 0 \\
\hline \multirow[t]{5}{*}{ Desmoronamiento } & Suprimido & & 0 \\
\hline & Intermedio & & 0 \\
\hline & Codominante & $<35$ & 0 \\
\hline & Codominante & $\geq 35$ & 1 \\
\hline & Dominante & & 0 \\
\hline
\end{tabular}


Cuadro 3. Análisis de Chi-cuadrado para el discriminante "I" de la ecuación (1) del modelo de estimación del volumen aprovechable en pie.

Chi-square analysis for parameter "I" of model (1) for log under bark volume estimation.

\begin{tabular}{lccccc}
\hline Fase de crecimiento del árbol & $\mathrm{n}$ & $\begin{array}{c}\text { Volumen maderable } \\
\text { sin corteza=0 } \\
\text { Real (\%) }\end{array}$ & $\begin{array}{c}\text { Volumen maderable } \\
\text { sin corteza }=0 \\
\text { Estimado (\%) }\end{array}$ & Chi-cuadrado & Significancia $P$ \\
\hline Crecimiento óptimo inicial & 84 & 95,2 & 95,2 & 0,131 & $<0,05$ \\
Crecimiento óptimo final & 417 & 59,0 & 56,1 & 0,594 & $<0,05$ \\
Envejecimiento & 748 & 47,1 & 48,8 & 0,386 & $<0,05$ \\
Desmoronamiento & 103 & 86,4 & 94,2 & 2,713 & $<0,05$ \\
\hline \multicolumn{1}{c}{ Clase social } & & & & \\
\hline Suprimido & 276 & 97,8 & 98,2 & 0,000 & $<0,05$ \\
Intermedio & 298 & 61,7 & 63,1 & 0,064 & $<0,05$ \\
Codominante & 605 & 39,2 & 39,5 & 0,003 & $<0,05$ \\
Dominante & 173 & 43,9 & 45,1 & 0,012 & $<0,05$ \\
\hline
\end{tabular}

Cuadro 4. Parámetros y estadísticos del componente " $\mathrm{M}$ " del modelo de estimación del volumen aprovechable en pie para diferentes sistemas de aprovechamiento.

Parameter "M" for log under bark volume model in three logging systems.

\begin{tabular}{cccccccccc}
\hline Modelo & $\mathrm{a}$ & $\mathrm{IC}$ inf & IC sup & $\mathrm{b}$ & $\mathrm{IC}$ inf & IC sup & $\mathrm{R}^{2} \mathrm{aj}$ & $\mathrm{n}$ & $\mathrm{MAE}\left(\mathrm{m}^{3}\right)$ \\
\hline VMSC F & 0,000436841 & 0,0002563 & 0,0006173 & 2,03513 & 1,92699 & 2,14328 & 70,72 & 338 & 0,2220 \\
VMSC TC & 0,00000667144 & 0,000001484 & 0,00001186 & 2,9975 & 2,82926 & 3,16573 & 80,56 & 128 & 0,1810 \\
VMSC TL & 0,0000123267 & $-5,172 \mathrm{E}-8$ & 0,00002470 & 2,83064 & 2,60983 & 3,05144 & 68,24 & 113 & 0,2664 \\
\hline
\end{tabular}

a, b: parámetros del modelo; IC: Intervalo de confianza al $95 \%$; inf: inferior; sup: superior; $\mathrm{R}^{2}$ aj: $\mathrm{R}^{2}$ ajustado significancia $95 \%$; MAE: error absoluto promedio; $\mathrm{F}=$ tratamiento de fustes completos; $\mathrm{TC}=$ tratamiento de trozas cortas; $\mathrm{TL}=$ tratamiento de trozas largas; $\mathrm{VMSC}=$ volumen maderable sin corteza.

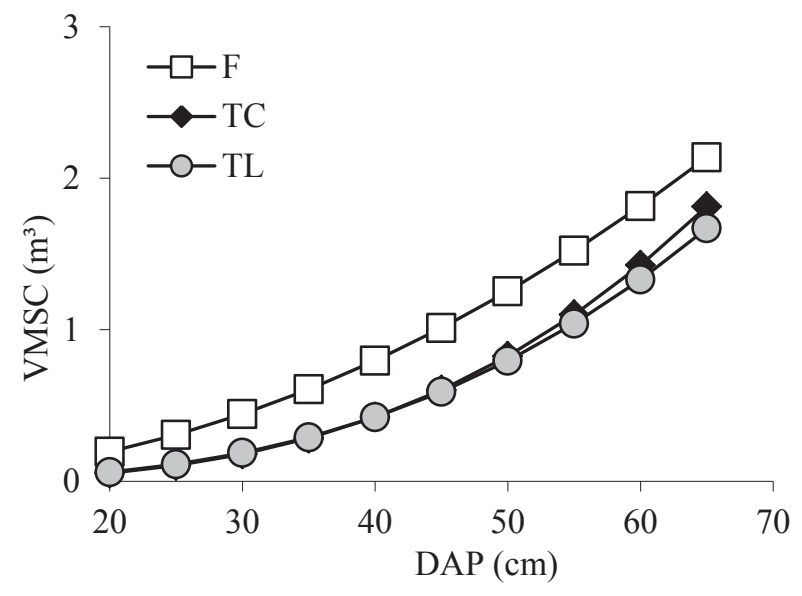

Figura 1. Modelo de estimación del volumen aprovechable de árboles maderables para diferentes sistemas de aprovechamiento; VMSC $=$ volumen maderable sin corteza

Log under bark volume model obtained for each logging system. VMSC $=\log$ under bark volume.
Caracterización de los volúmenes para los diferentes tipos de corta y sistemas de aprovechamiento. En los volúmenes totales iniciales no se encontraron diferencias significativas entre los tratamientos ensayados $\left(726,5\right.$ a $\left.850,3 \mathrm{~m}^{3} \mathrm{ha}^{-1}\right)$ (cuadro 5), siendo la relación volumen/área basal prácticamente constante $\left(11,8\right.$ a $\left.12,1 \mathrm{~m}^{3} \mathrm{~m}^{-2}\right)$. Al analizar el volumen total aprovechado mediante floreo, el sistema de fustes completos difirió significativamente de los tratamientos de troza corta y troza larga, con un rendimiento más del doble en el aprovechamiento del bosque. Los índices de aprovechamiento obtenidos fueron de $45,5 \%$ para fustes completos, $21,3 \%$ para troza larga y $22,4 \%$ para troza corta. En cuanto al volumen maderable, el rendimiento de fustes completos representó el $64,2 \%$ del volumen total apeado, en comparación con $29 \%$ y $33 \%$ de troza corta y troza larga, respectivamente. Para los volúmenes de astilla y desecho no se encontraron diferencias significativas entre tratamientos.

Se observa un aumento en el VMSC al completar la corta de protección en los tratamientos de troza corta $\left(27,0 \mathrm{~m}^{3} \mathrm{ha}^{-1}\right)$ y troza larga $\left(16,8 \mathrm{~m}^{3} \mathrm{ha}^{-1}\right)$, mientras que en el aprovecha- 
Cuadro 5. Evolución del volumen (total, maderable, astillable y desecho) durante la corta de protección.

Total over bark volume, log under bark volume, log for chips production volume and wood residue volume in the shelterwood cut system of the studied plots.

\begin{tabular}{clccc}
\hline Tratamiento & \multicolumn{1}{c}{ VTCC } & VMSC & VASC & VD \\
\hline BSITC & $726,5(12,1)$ & $99,9 \mathrm{a}(1,7 \mathrm{a})$ & $360,8 \mathrm{ab}(6,0 \mathrm{a})$ & $265,7(3,5 \mathrm{a})$ \\
BSITL & $750,4(11,9)$ & $92,9 \mathrm{a}(1,4 \mathrm{a})$ & $409,8 \mathrm{a}(6,5 \mathrm{a})$ & $247,7(3,9 \mathrm{ab})$ \\
BSIF & $850,3(11,8)$ & $342,2 \mathrm{~b}(4,8 \mathrm{~b})$ & $265,2 \mathrm{~b}(3,7 \mathrm{~b})$ & $242,8(3,4 \mathrm{~b})$ \\
FLTC & $162,5 \mathrm{a}(11,8 \mathrm{a})$ & $47,1 \mathrm{a}(3,3 \mathrm{a})$ & $57,1(4,3 \mathrm{a})$ & $58,3(4,2 \mathrm{a})$ \\
FLTL & $159,9 \mathrm{a}(11,0 \mathrm{ab})$ & $53,4 \mathrm{a}(3,5 \mathrm{a})$ & $58,1(4,2 \mathrm{a})$ & $48,3(3,3 \mathrm{a})$ \\
FLF & $387,1 \mathrm{~b}(10,6 \mathrm{~b})$ & $248,1 \mathrm{~b}(6,8 \mathrm{~b})$ & $66,3(1,9 \mathrm{~b})$ & $72,7(1,9 \mathrm{~b})$ \\
CPTC & $411,3(11,6)$ & $74,1 \mathrm{a}(2,1 \mathrm{a})$ & $176,6 \mathrm{ab}(4,8 \mathrm{a})$ & $160,6(4,6)$ \\
CPTL & $440,5(11,4)$ & $70,2 \mathrm{a}(1,8 \mathrm{a})$ & $215,4 \mathrm{a}(5,6 \mathrm{a})$ & $154,9(4,0)$ \\
CPF & $487,4(11,3)$ & $255,9 \mathrm{~b}(5,9 \mathrm{~b})$ & $96,8 \mathrm{~b}(2,3 \mathrm{~b})$ & $134,7(3,1)$ \\
RTC & $315,2(13,0)$ & $25,9 \mathrm{a}(1,1 \mathrm{a})$ & $184,2(7,6 \mathrm{a})$ & $105,1(4,3 \mathrm{~b})$ \\
RTL & $309,9(12,6)$ & $22,7 \mathrm{a}(0,9 \mathrm{a})$ & $194,4(7,9 \mathrm{a})$ & $92,8(3,8 \mathrm{a})$ \\
RF & $362,9(12,5)$ & $86,4 \mathrm{~b}(3,0 \mathrm{~b})$ & $168,4(5,8 \mathrm{~b})$ & $108,1(3,7 \mathrm{a})$ \\
\hline
\end{tabular}

Entre paréntesis se presentan las relaciones volumen/área basal $\left(\mathrm{m}^{3} \mathrm{~m}^{-2}\right)$; BSITC $=$ situación inicial tratamiento de trozas cortas; BSITL $=$ situación inicial tratamiento de trozas largas; BSIF = situación inicial tratamiento de fustes completos; FLTC = floreo tratamiento de trozas cortas; FLTL = floreo tratamiento de trozas largas; FLF = floreo tratamiento de fustes completos; $\mathrm{CPTC}=$ corta de protección tratamiento de trozas cortas; $\mathrm{CPTL}=\mathrm{corta}$ de protección tratamiento de trozas largas; $\mathrm{CPF}=$ corta de protección tratamiento de fustes completos; RTC = bosque remanente tratamiento de trozas cortas; RTL = bosque remanente tratamiento de trozas largas; $\mathrm{RF}=$ bosque remanente tratamiento de fustes completos; VTCC $=$ volumen total con corteza $\left(\mathrm{m}^{3} \mathrm{ha}^{-1}\right)$; VMSC $=$ volumen maderable sin corteza $\left(\mathrm{m}^{3} \mathrm{ha}^{-1}\right) ; \mathrm{VASC}=$ volumen de astillas sin corteza $\left(\mathrm{m}^{3}\right.$ ha $\left.{ }^{-1}\right)$; VD = volumen de desecho con corteza $\left(\mathrm{m}^{3} \mathrm{ha}^{-1}\right)$. En cada columna, letras diferentes indican diferencias significativas $(P<0,01)$.

miento de fustes completos no varió grandemente $\left(7,8 \mathrm{~m}^{3}\right.$ $\left.\mathrm{ha}^{-1}\right)$. Los volúmenes aprovechados (VMSC) presentaron diferencias significativas para el tratamiento de fuste completo (52,5\% del VTCC comparado con $18 \%$ y $16 \%$ de troza corta y troza larga, respectivamente) partiendo de similares volúmenes de cosecha. Se observó que existe un aumento en el volumen de astillas en los tratamientos troza corta y troza larga, manteniéndose sin diferencias el volumen de desecho entre tratamientos. La gran cantidad de volumen maderable de fuste completo se puede deducir como un aporte de madera que se produce desde el volumen astillable. Esto se debe a que se incluyeron un mayor número de trozas de bajo diámetro, en contraposición con los otros tratamientos que subutilizan el volumen maderable (figura 2). El número de trozas y el volumen maderable producido mediante el aprovechamiento de fustes completos, se incrementa para las clases de diámetro $25-30 \mathrm{~cm}$ hasta 40-45 $\mathrm{cm}$. En el tratamiento de trozas cortas se obtuvieron 115 trozas de $0,64 \mathrm{~m}^{3}$ cada uno de volumen medio, mientras que en tratamiento de fustes completos se aprovecharon 710 trozas de $0,38 \mathrm{~m}^{3}$ cada una.

Las relaciones de volumen aprovechado/área basal extraída presentaron diferencias significativas entre los tratamientos ensayados. Las relaciones con volumen maderable fueron máximas durante el floreo $\left(3,3 \mathrm{a} 6,8 \mathrm{~m}^{3} \mathrm{~m}^{-2}\right)$, disminuyendo al completar la corta de protección $(1,8 \mathrm{a}$
$5,9 \mathrm{~m}^{3} \mathrm{~m}^{-2}$ ). En forma inversa, los tratamientos de troza corta y troza larga presentaron relaciones de volumen astillable/área basal extraída más grandes $\left(4,2\right.$ a $\left.5,6 \mathrm{~m}^{3} \mathrm{~m}^{-2}\right)$ que en el tratamiento de fustes $\left(2 \mathrm{~m}^{3} \mathrm{~m}^{-2}\right)$ (cuadro 5). El tratamiento de fustes completo maximizó el aprovechamiento de los individuos durante el floreo, no incrementando significativamente los volúmenes maderables al completar la corta de protección, pero incrementándose considerablemente los índices de desecho/área basal cortada $\left(1,9 \mathrm{~m}^{3} \mathrm{~m}^{-2}\right.$ para el floreo $3,1 \mathrm{~m}^{3} \mathrm{~m}^{-2}$ en la corta de protección).

\section{DISCUSIÓN}

El número de trozas y el volumen maderable producido mediante el aprovechamiento de fustes completos se incrementa para las clases de diámetro $25-30 \mathrm{~cm}$ hasta $40-45 \mathrm{~cm}$. Este volumen representa valores muy importantes de madera sana que usualmente queda en el bosque o en pie, siendo despreciado por poseer bajo volumen individual de troza. Cabe considerar que el pago a los motosierristas se realiza por troza (raramente por volumen), por lo que los contratistas exigen trozas grandes de buena calidad.

Los estudios de rendimientos volumétricos de aprovechamientos forestales en bosques de $N$. pumilio pertenecen a toda la gama de clases de sitio, variando sus volúmenes totales entre 378,2 y $945,3 \mathrm{~m}^{3}$ ha $^{-1}$ y sus relaciones volu- 

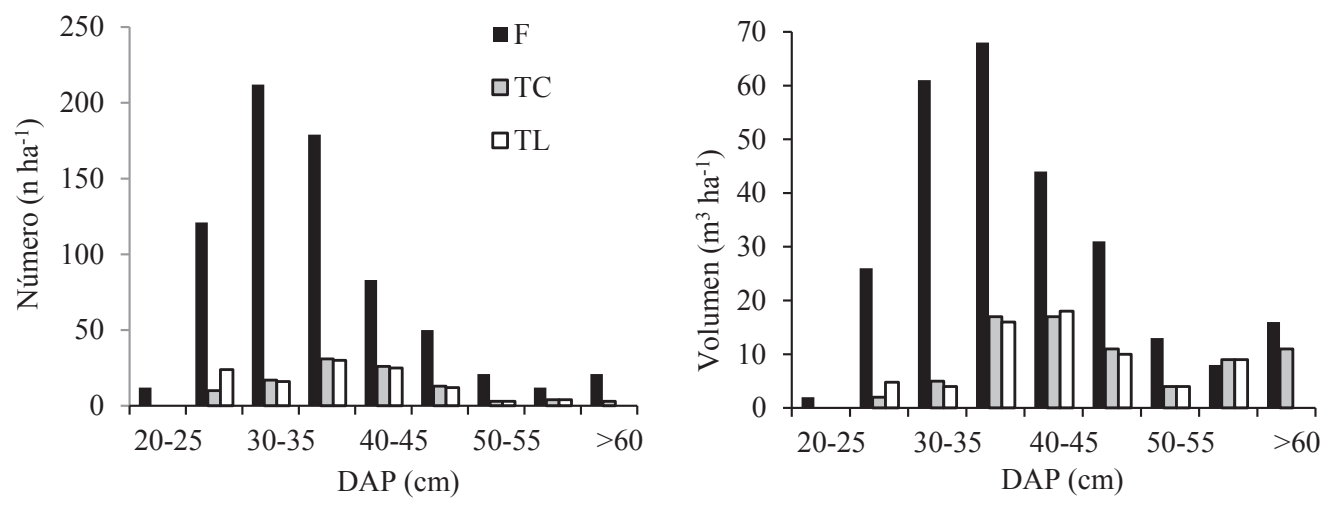

Figura 2. Comparación del número y volumen de trozas por clases de diámetro en punta fina para los tratamientos de aprovechamiento de troza corta (TC), troza larga (TL) y fuste completo (F).

Number and volume of logs according to diameter class and logging system (short-logs (TC), long-logs (TL) and complete stems (F)).

men total/área basal entre 8,91 y $14,03 \mathrm{~m}^{3} \mathrm{~m}^{-2}$, presentando volúmenes obtenidos en una corta de protección que varían de acuerdo a diferentes autores, entre 33,0 y 266,5 $\mathrm{m}^{3} \mathrm{ha}^{-1}$ (Chauchard 1990, Ferrando 1994, Bava y Hlopec 1995, González 1995, Mosqueda 1995, Garib 1996, Daffunchio y Villena 1997, Martínez Pastur et al. 2004).

Los valores obtenidos por González (1995), Garib (1996) y Daffunchio y Villena (1997) son comparables a los obtenidos en las parcelas de fuste completo, habiéndose utilizado sistemas de aprovechamiento similares. Los índices de cosecha obtenidos variaron entre un $20 \%$ y un $35 \%$, lo que implica haber maximizado la utilización de los árboles apeados. Estos índices de cosecha representan entre 3,5 y 7,6 $\mathrm{m}^{3} \mathrm{~m}^{-2}$ de volumen maderable/área basal. Los resultados obtenidos por Ferrando (1994) en un bosque mixto de $N$. pumilio- $N$. dombeyi (altura dominante de 24 a $25 \mathrm{~m}$ ) son comparables a los rendimientos de los tratamientos troza corta y troza larga (índice de cosecha de 13,2 \%). Los índices de cosecha presentados en el cuadro 6 para aprovechamientos mediante floreos o raleos varían entre $1,5 \%$ y $11,2 \%$, sin considerar el tratamiento de fuste completo. La metodología de fuste completo fue aplicada a gran escala en la estancia Los Cerros (Tierra del Fuego, Argentina) (Martínez Pastur et al. 2004) dando resultados comparables a los de este estudio. Se obtuvieron valores de 177,4 y $167,0 \mathrm{~m}^{3} \mathrm{ha}^{-1}$ de volumen maderable sin corteza (cuadro 6) empleando el método de regeneración de Retención Variable (Martínez Pastur y Lencinas 2005, Vergara y Schlatter 2006, Martínez Pastur et al. 2007b, Lencinas et al. 2007, 2008). Los autores manifiestan que los volúmenes extraídos fueron altos ya que durante las faenas en la producción de fustes no se realizaron saneamientos innecesarios.

El sistema de fuste completo incrementa el rendimiento volumétrico del bosque comparado con los métodos de trozas cortas y trozas largas, produciendo otras ventajas como un menor impacto en el suelo debido a la menor cantidad de recorrido realizado por la maquinaria forestal (Martínez Pastur et al. 2004, 2007, Cellini et al. 2005), siendo importante para disminuir el daño a la regeneración pre instalada (Saveneh y Dignan 1997, Martínez Pastur et al. 1999, Rosenfeld et al. 2006) no dimensionada en este estudio. Un efecto no deseado es el daño a los arboles remanentes durante las tareas de aprovechamiento (Hickey et al. 2001), que producen la caída de los arboles remanentes por volteo de viento después de la cosecha (GeaIzquierdo et al. 2004), siendo necesario realizar estas tareas con sumo cuidado para reducir daños en los arboles remanentes (Hickey et al. 2001). Asimismo, este sistema, debido a sus altos rendimientos, permite el aprovechamiento de bosques en las peores calidades de sitio (Martínez Pastur et al. 2000). Estos sistemas de aprovechamiento poseen implicancias en la exportación de nutrientes desde el bosque, siendo importante dejar en el terreno todo el componente hojas, ramas finas y corteza, por ser estos los que presentan mayor concentración de nutrientes (Wang et al. 1996, Goya et al. 2003, Gargaglione et al. 2013). Asimismo, la conservación de árboles remanentes en el método de regeneración empleado, presenta beneficios en términos de estructura de hábitat para pájaros (Lencinas et al. 2009), musgos (Lencinas et al. 2008), plantas del sotobosque (Lencinas et al. 2011) e insectos (Lencinas et al. 2014), siendo estos aspectos muy tenidos en cuenta en la planificación del uso de los territorios. En este estudio se presentan los resultados desde un punto de vista de producción, siendo de suma importancia para futuras investigaciones los aspectos ambientales y sociales.

\section{CONCLUSIONES}

Realizando un floreo del bosque se logra un menor rendimiento en volumen maderable en comparación con la corta de protección, ya que existe un alto volumen de trozas de diámetro pequeño que quedan en el bosque junto a la 
Cuadro 6. Comparación de rendimientos volumétricos en diferentes bosques de N. pumilio.

Timber volume obtained in shelterwood cuts along $N$. pumilio forests.

\begin{tabular}{|c|c|c|c|c|c|c|}
\hline Zona & BSI VTCC & BSI VTCC/AB & R VMSC & F VMSC & CP VMSC & $\mathrm{VMSC} / \mathrm{AB}$ \\
\hline $\mathrm{TC}$ & 726,5 & 12,17 & -- & $47,1(6,5)$ & $74,1(10,2)$ & $2,08(\mathrm{CP})$ \\
\hline $\mathrm{TL}$ & 750,4 & 11,84 & -- & $53,4(7,1)$ & $70,2(9,3)$ & $1,81(\mathrm{CP})$ \\
\hline $\mathrm{F}$ & 850,3 & 11,6 & -- & $248,1(29,2)$ & $255,9(30,1)$ & $5,96(\mathrm{CP})$ \\
\hline Russfin $^{a}$ & 945,3 & 12,53 & -- & -- & $190,3(20,1)$ & 3,83 \\
\hline MonteAlto ${ }^{\mathrm{b}}$ & 776,2 & 9,28 & -- & $87,3(11,2)$ & $166,7(21,5)$ & $3,52(\mathrm{CP})$ \\
\hline Aysén ${ }^{c}$ & 547,6 & 11,48 & -- & $60,3(11,0)$ & $72,5(13,2)$ & $2,63(\mathrm{CP})$ \\
\hline MonteAlto $^{\mathrm{d}}$ & 633,7 & 8,91 & $39,8(6,3 \%)$ & -- & -- & 2,66 \\
\hline MonteAlto $^{\mathrm{e}}$ & 552,7 & 9,10 & $44,3(8,0 \%)$ & -- & -- & 4,53 \\
\hline Tolhuin $^{f}$ & 586,9 & 10,01 & -- & -- & $179,7(30,6)$ & 5,86 \\
\hline MaCristina $^{\mathrm{f}}$ & 535,1 & 10,53 & -- & -- & $152,1(28,4)$ & 4,14 \\
\hline PretoOeste $^{\mathrm{f}}$ & 750,2 & 10,41 & -- & -- & $266,5(35,5)$ & 5,69 \\
\hline Cholilag $^{g}$ & 378,2 & 14,03 & -- & $16,8(4,4)$ & -- & 3,66 \\
\hline Cholilag $^{g}$ & 582,1 & 9,76 & -- & $8,8(1,5)$ & -- & 0,91 \\
\hline Cholila $^{\mathrm{g}}$ & 756,5 & 14,03 & -- & $82,4(10,9)$ & -- & 4,85 \\
\hline Tolhuin $^{\mathrm{h}}$ & 659,0 & 10,73 & -- & -- & $33,0(5,0)$ & -- \\
\hline Los Cerros ${ }^{\mathrm{i}}$ & 534,9 & 11,88 & -- & -- & $177,4(33,2)$ & 5,91 \\
\hline Los Cerros $^{\mathrm{i}}$ & 449,5 & 11,25 & -- & -- & $167,0(37,1)$ & 5,57 \\
\hline
\end{tabular}

Entre paréntesis se presentan los índices de cosecha obtenidos en porcentaje; BSI VTCC = situación inicial volumen total con corteza $\left(\mathrm{m}^{3}\right.$ ha $\left.{ }^{-1}\right)$; BSI $\mathrm{VTCC} / \mathrm{AB}=$ situación inicial relación volumen total con corteza / área basal $\left(\mathrm{m}^{3} \mathrm{~m}^{-2}\right) ; \mathrm{R}$ VMSC $=$ raleo volumen maderable sin corteza $\left(\mathrm{m}^{3}\right.$ ha $\left.{ }^{-1}\right) ; \mathrm{F}$ $\mathrm{VMSC}=$ floreo volumen maderable sin corteza $\left(\mathrm{m}^{3} \mathrm{ha}^{-1}\right) ; \mathrm{CP}$ VMSC $=$ corta de protección volumen maderable sin corteza $\left(\mathrm{m}^{3}\right.$ ha- $\left.\mathrm{C}^{-1}\right) ; \mathrm{TC}=$ tratamiento de trozas cortas San Justo; TL = tratamiento de trozas largas San Justo; F = tratamiento de fustes completos San Justo; (a) Tierra del Fuego (Chile) en Garib (1996); (b) XII Región (Chile) en González (1995); (c) XI Región (Chile) en Ferrando (1994); (d) rodal en crecimiento óptimo avanzado, XII Región (Chile) en Mosqueda (1995); (e) rodal en crecimiento óptimo final, XII Región (Chile) en Mosqueda (1995); (f) Tierra del Fuego (Argentina) en Daffunchio y Villena (1997); (g) Chubut (Argentina) en Chauchard (1990); (h) Tierra del Fuego (Argentina) en Bava y Hlopec (1995); (i) Tierra del Fuego (Argentina) en Martínez Pastur et al. (2004).

copa o en pie. Por otra parte, existe un beneficio adicional al transformar el bosque en un sistema regular con todas las ventajas que ello implica. El método de fustes completos fue el que dio mejores resultados en comparación con los de extracción por medio de trozas ya que incrementa el rendimiento volumétrico del bosque, y presenta el menor impacto en el suelo. Sin embargo, se debe tener especial cuidado con el daño producido durante las tareas de aprovechamiento a los árboles remanentes, que producen la inestabilidad del bosque de protección. Los rendimientos obtenidos en este estudio son comparables a estudios realizados en Argentina y Chile siendo posibles de aplicar a gran escala sin la necesidad de producir astillas y aprovechar bosques de baja calidad de sitio. Considerar nuevas alternativas en la planificación del aprovechamiento dentro del manejo forestal de los bosques permite obtener un mayor índice de cosecha, aumentando los beneficios para la empresa forestal y disminuyendo los requerimientos de superficie de bosques dentro del tranzón.

\section{AGRADECIMIENTOS}

A Douglas Sharpe, Guillermina Dalla Salda, Gabriela Staffieri, Victoria Skrt, Paula Ferrere y Cristian Goldman por su desinteresada participación en el trabajo; y al aserradero Los Castores, al Centro Austral de Investigaciones Científicas y Técnicas, la Universidad Nacional de la Patagonia Austral y el Instituto Nacional de Tecnología Agropecuaria por el apoyo institucional y financiero.

\section{REFERENCIAS}

Bava J, R Hlopec. 1995. El manejo sustentable de la Lenga en Tierra del Fuego. In Actas IV Jornadas Forestales Patagónicas. San Martín de Los Andes, 24-27 octubre. p. 81-96.

Cailliez F. 1980. Estimación del volumen forestal y predicción del rendimiento con referencia especial a los trópicos. Vol. 1 - estimación del volumen. Estudio FAO 22/1. 80 p.

Cellini JM, G Martínez Pastur, R Vukasovič, MV Lencinas, B 
Díaz, E Wabö. 2005. Pautas de sustentabilidad en el manejo forestal de los bosques de Nothofagus pumilio (Poepp. et Endl.) Krasser. Yvyraretá 13: 77-82.

Chauchard, LM. 1990. Plan de ordenación del cuartel Lomadas de Cholila, Provincia de Chubut. Proyecto de desarrollo forestal del Área Cordillerana. Buenos Aires, Argentina. Consejo Federal de Inversiones. $50 \mathrm{p}$.

Clutter JL, JC Fortson, LV Pienaar, GH Brister, RL Bailey. 1983. Timber Management: A Cuantitative Approach. New York, USA. John Wiley. 333 p.

Collado L. 2001. Los bosques de Tierra del Fuego. Análisis de su estratificación mediante imágenes satelitales para el inventario forestal de la provincia. Multequina 10: 1-16.

Cordone VJ, J Bava. 1997. Aplicación de la clasificación de madera en rollo de Lenga. Esquel, Argentina. CIEFAP. 32 p. (Publicación técnica $\mathrm{N}^{\circ} 26$ )

Cremer KW, CJ Borough, FH Mckinnell, PR Carter. 1982. Effects of stocking and thinning on wind damage in plantations. New Zealand Journal of Forestry Science 12(2): 244-268.

Daffunchio I, P Villena. 1997. Informe final de las Áreas Piloto de Rendimiento de Lenga Patagonia SA. Río Grande, Argentina. Lenga Patagonia S.A. 34 p.

Ferrando M. 1994. Estructura y rendimientos volumétricos bajo corta de protección de un bosque de Lenga en Aysén, XI Región. Tesis Ingeniero Forestal. Santiago, Chile. Universidad de Chile. 53 p.

Gargaglione V, PL Peri, G Rubio. 2013. Partición diferencial de nutrientes en árboles de Nothofagus antarctica creciendo en un gradiente de calidades de sitio en Patagonia Sur. Bosque 34(3): 291-302. DOI: 10.4067/S0717-92002013000300005

Garib IA. 1996. Rendimientos volumétricos en bosque de lenga (Nothofagus pumilio) sometidos a cortas de protección. Provincia de Tierra del Fuego, XII Región. Tesis Ingeniero Forestal. Santiago, Chile. Universidad de Chile. 55 p.

Gea-Izquierdo G, G Martınez Pastur, JM Cellini, MV Lencinas. 2004. Forty years of silvicultural management in southern Nothofagus pumilio (Poepp. et Endl) Krasser primary forests. Forest Ecology and Management 201(2-3): 335-347. doi:10.1016/j.foreco.2004.07.015

González SA. 1995. Rendimientos volumétricos en la corta de protección de un bosque de lenga en Monte Alto, XII Región. Tesis Ingeniero Forestal. Santiago, Chile. Universidad de Chile. 51 p.

Goya JF, C Pérez, JL Frangi, R Fernandez. 2003. Impacto de la cosecha y destino de los residuos sobre la estabilidad del capital de nutrientes en plantaciones de Pinus taeda L. Ecología Austral 13: 139-150.

Hickey JE, MG Neyland, OD Bassett. 2001. Rationale and design for the Warra silvicultural systems trial in wet Eucalyptus obliqua forests in Tasmania. Tasforests 13(2): 155-182.

Husch B, TW Beers, JA Kershaw. 2003. Forest Mensuration, Fourth edition. Malabar, USA. Krieger Publishing. 443 p.

Lencinas MV, G Martínez Pastur, EA Gallo, A Moretto, C Busso, P Peri. 2007. Mitigation of biodiversity loss in Nothofagus pumilio managed forests of South Patagonia. In Pacha MJ, S Luque, L Galetto, L Iverson eds. Understanding Biodiversity Loss: An Overview of Forest Fragmentation in South America. Part III. Landscape Ecology for Conservation, Management and Restoration. IALE Landscape Research and Management Papers. p. 112-120.

Lencinas MV, G Martínez Pastur, R Solan, EA Gallo, JM Cellini.
2008. Forest management with variable retention impact over bryophyte communities of Nothofagus pumilio understory. Forstarchiv 79: 77-82.

Lencinas MV, G Martínez Pastur, EA Gallo, JM Cellini. 2009. Alternative silvicultural practices with variable retention improve bird conservation in managed South Patagonian forests. Forest Ecology and Management 258: 472-480. doi:10.1016/j.foreco.2009.01.012

Lencinas MV, G Martínez Pastur, EA Gallo, JM Cellini. 2011. Alternative silvicultural practices with variable retention to improve understory plant diversity conservation in southern Patagonian forests. Forest Ecology and Management 262: 1236-1250

Lencinas MV, G Martínez Pastur, E Gallo, JM Cellini. 2014. Decreasing negative impacts of harvesting over insect communities using variable retention in southern Patagonian forests. Journal of Insect Conservation 18: 479-495. DOI 10.1007/s10841-014-9661-5

Marquardt DW. 1963. An algorithm for least squares estimation of nonlinear parameters. Journal of the Society for Industrial and Applied Mathematics 11(2): 431-441.

Martínez Pastur G, PL Peri, R Vukasovic, S Vaccaro, V Piriz Carrillo. 1997. Site index equation for Nothofagus pumilio Patagonian forest. Phyton 61(1/2): 55-60.

Martínez Pastur G, PL Peri, MC Fernández, G Staffieri. 1999. Desarrollo de la regeneración a lo largo del ciclo del manejo forestal de un bosque de Nothofagus pumilio: Incidencia de la cobertura y el aprovechamiento o cosecha. Bosque 20(2): 39-46.

Martínez Pastur G, JM Cellini, PL Peri, R Vukasovic, C Fernandez. 2000. Timber production of Nothofagus pumilio forests by a shelterwood system in Tierra del Fuego (Argentina). Forest Ecology and Management 134(1-3): 153-162.

Martínez Pastur G, JM Cellini, MV Lencinas, B Díaz, PL Peri, R Vukasovic. 2001-2002. Funciones de rendimiento volumétrico en pie y en aserradero para la lenga (Nothofagus pumilio). Ciencias Forestales 15(1-2): 32-45.

Martínez Pastur G, JM Cellini, RF Vukasovic. 2004. Informe de rendimiento del Aserradero Kareken. In Secretaría de Ambiente y Desarrollo Sustentable de la Nación, Dirección de Bosques ed. Informe Final: Subproyecto "Aplicación a gran escala en un aserradero medio". PIARFON. Proyecto BIRF 4085-AR (PNUD - Banco Mundial). p. 319-352.

Martínez Pastur G. 2006. Biometría y Producción Forestal para bosques naturales de Nothofagus pumilio en Tierra del Fuego. Tesis Doctoral. Bahía Blanca, Argentina. Facultad de Agronomía, Universidad Nacional del Sur. 242 p.

Martínez Pastur G, MV Lencinas. 2005. El manejo forestal en los bosques de Nothofagus pumilio en Tierra del Fuego. IDIAXXI 5(8): 107-110.

Martínez Pastur G, MV Lencinas, PL Peri, M Arena. 2007a. Photosynthetic plasticity of Nothofagus pumilio seedlings to light intensity and soil moisture. Forest Ecology and Management 243(2): 274-282.

Martínez Pastur G, MV Lencinas, P Peri, A Moretto, JM Cellini, I Mormeneo, R Vukasovic. 2007b. Harvesting adaptation to biodiversity conservation in sawmill industry: technology innovation and monitoring program. Journal of Technology Management \& Innovation 2(3): 58-70.

Martínez Pastur G, MV Lencinas, JM Cellini, PL Peri, R Soler Esteban. 2009. Timber management with variable retention in Nothofagus pumilio forests of Southern Patagonia. Fo- 
rest Ecology and Management 258(4): 436-443.

Martínez Pastur G, JM Cellini, MV Lencinas, M Barrera, PL Peri. 2011. Environmental variables influencing regeneration of Nothofagus pumilio in a system with combined aggregated and dispersed retention. Forest Ecology and Management 261: 178-186.

Mosqueda C. 1995. Rendimientos volumétricos en el raleo de un bosque de lenga (Nothofagus pumilio) en la XII Región. Tesis Ingeniero Forestal. Santiago, Chile. Universidad de Chile. $68 \mathrm{p}$.

Rosenfeld JM, RM Navarro Cerrillo, JR Guzman Alvarez. 2006. Regeneration of Nothofagus pumilio (Poepp. et Endl) Krasser forests after five years of seed tree cutting. Journal of Environmental Management 78: 44-51.

Saveneh A, P Dignan. 1997. The use of shelterwood in Eucalyptus regnans forest: the effect of overwood removal at three years on regeneration stocking and health. Australian Forestry 60(4): 251-259.

Schmidt H, A Urzúa. 1982. Transformación y manejo de los bosques de lenga en Magallanes. Santiago, Chile. Universidad de Chile. 62 p. (Ciencias Agrícolas $\mathrm{N}^{\circ} 11$ )

Vergara PM, RP Schlatter. 2006. Aggregate retention in two Tierra del Fuego Nothofagus forests: short-term effect on bird abundance. Forest Ecology and Management 225: 213-224.

Wang JR, AL Zhong, SW Simard, JP Kimmins. 1996. Aboveground biomass and nutrient accumulation in an age sequence of paper birch (Betula papyrifera) in the Interior Cedar Hemlock zone, British Columbia. Forest Ecology and Management 83: 27-38.

Wilson JS, CD Oliver. 2000. Stability and density management in Douglas-fir plantations. Canadian Journal of Forest Research 30: 910-920.

Recibido: 20.10 .15

Aceptado: 18.08 .17 
